\title{
The Temperature and Cooling Age of the White-Dwarf Companion to the Millisecond Pulsar PSR B1855+09
}

\author{
Jon Bell \\ ATNF, CSIRO, PO Box 76 Epping NSW 1710 AUSTRALIA; \\ jbell@atnf.csiro.au \\ Marten van Kerkwijk, Utrecht, M.H.vanKerkwijk@astro.uu.nl \\ Vicky Kaspi, MIT, vicky@space.mit.edu \\ Shri Kulkarni, CalTech, srk@astro.caltech.edu
}

\begin{abstract}
We report on Keck and HST observations of the binary millisecond pulsar PSR B1855+09. We detect its white-dwarf companion and measure $m_{\mathrm{F} 555 \mathrm{~W}}=25.90 \pm 0.12$ and $m_{\mathrm{F} 814 \mathrm{~W}}=24.19 \pm 0.11$ (Vega system). From the reddening-corrected color we infer a temperature $T_{\text {eff }}=4800 \pm 800 \mathrm{~K}$. The companion mass is known accurately from measurements of the Shapiro delay of the pulsar signal, $M_{\mathrm{C}}=0.258_{-0.016}^{+0.028} \mathrm{M}_{\odot}$. Given a cooling model, one can use the measured temperature to determine the cooling age. The main uncertainty in the cooling models for such low-mass white dwarfs is the amount of residual nuclear burning, which depends on the thickness of the hydrogen layer surrounding the helium core. For PSR B1855+09, such models lead to a cooling age of $\sim 10 \mathrm{Gyr}$, which is twice the spin-down age of the pulsar. It may be that the pulsar does not brake $(n=3.0)$ like a dipole rotating in vacuo. For other pulsar companions, however, ages well over $10 \mathrm{Gyr}$ are inferred, indicating that the problem may lie with the cooling models. There is no age discrepancy for models in which the white dwarfs are formed with thinner hydrogen layers $\left(<3 \times 10^{-4} \mathrm{M}_{\odot}\right)$. See van Kerkwijk et al. ApJ (submitted) for more details.
\end{abstract}

\section{Discussion}

The white dwarf age inferred from the Driebe et al. (1998, A\&A, 339, 123) model is greater than the characteristic age, $\tau_{\mathrm{c}}=5 \mathrm{Gyr}$. If the model were correct, $\tau_{c}$ must be an underestimate of the true age $t_{\mathrm{PSR}}$ of the PSR B1855+09 system. One interpretation would be that the braking index of a millisecond pulsar is less than the canonical value of 3 ; to obtain $t_{\mathrm{PSR}}>8 \mathrm{Gyr}$ would require $n<2.25$. This is perhaps not unreasonable, as for most pulsars for which braking indices could be measured, values less than 3 have been found. We note that a variant on the vacuum-dipole model (Melatos 1997, 288, 1049), 


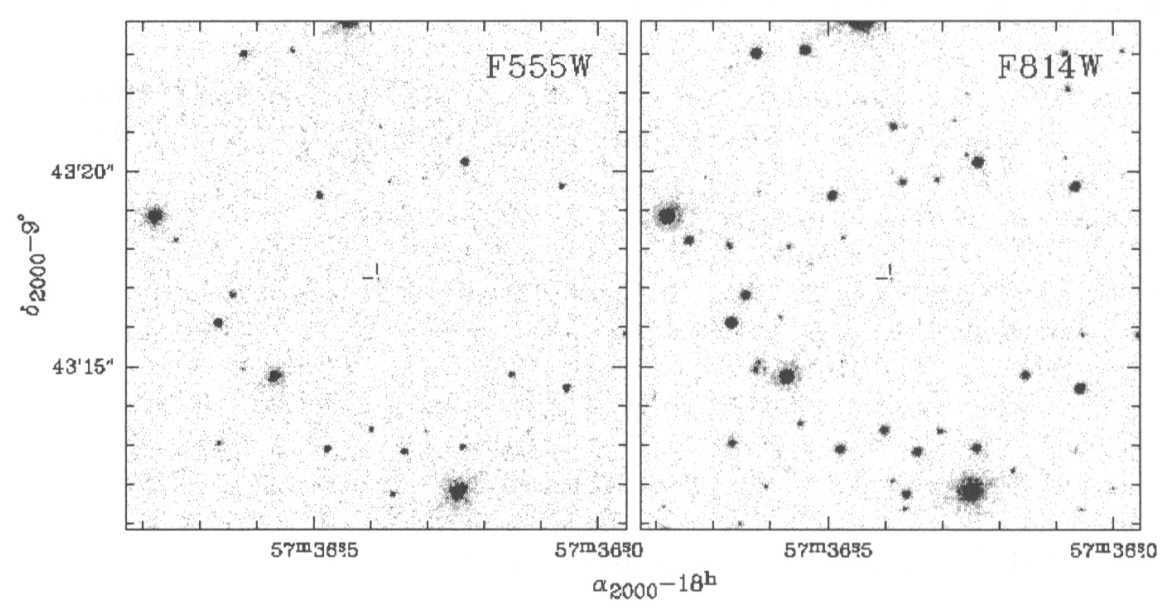

Figure 1. HST images of the PSR B1855+09 field, taken through the $\mathrm{F} 555 \mathrm{~W}$ and $\mathrm{F} 814 \mathrm{~W}$ filters. The 1998.0 timing position is shown by the tick marks. These are 0.24 long, equal to the $95 \%$ confidence diameter inferred from the uncertainty in the astrometric tie.

which does a reasonable job of explaining these braking indices, predicts $n=3$ for a pulsar like PSR B1855+09.

The optical counterparts of other pulsar binaries may give a clue to where the problem lies. From the list of temperatures compiled by Hansen \& Phinney (1998, MNRAS, 294, 569), we find that two pulsars, PSR J0034-0534 and PSR J1713+0747, have very cool companions, with $T_{\text {eff }}<3500 \mathrm{~K}$ and $T_{\text {eff }}=$ $3400 \pm 300 \mathrm{~K}$, respectively. For such temperatures, the cooling ages inferred from the models of Driebe et al. are well over $10 \mathrm{Gyr}$, even if the orbital inclinations were such that the helium white dwarfs had close to the maximum mass. This suggests that the models may overestimate the cooling ages.

The above is in contrast to PSR J1012+5307, where the cooling age estimated using the Driebe et al. model is very similar to the characteristic age. The discrepancy might be resolved by the thickness of the hydrogen layer being a function of the orbital separation, perhaps via somewhat different mass-loss histories. PSR J1012+5307 has the second-shortest period of all systems known (0.6d), much shorter than that of PSR B1855+09 (12.3d). PSR J0034-0534, however, has a short orbital period $(1.6 \mathrm{~d})$ too. The discrepancy might also result from differences in white-dwarf mass. For instance, Driebe et al. (1999 in press) find that shell flashes only occur in a limited range of masses (0.21$0.30 \mathrm{M}_{\odot}$ ), which includes PSR B1855+09 but not PSR J1012+5307. Without accurate masses for the other systems, this hypothesis is hard to refute. Driebe et al., however, find that the flashes have only minimal effect on the cooling ages. Thus, for now this puzzle remains to be solved. 